VÂNIA MUNIZ NÉQUer SOARES ${ }^{1}$

KLeyde Ventura de SOUZA ${ }^{2}$

Tatiana Claumann Freygang ${ }^{3}$

VANeSSA CoRReA ${ }^{4}$

MARIA RIALTO SAITO

\section{Mortalidade materna por pré-eclâmpsia/ eclâmpsia em um estado do Sul do Brasil}

\author{
Maternal mortality due to pre-eclampsialeclampsia in a state in \\ Southern Brazil
}

Artigo original

Palavras-chave

Mortalidade materna Hipertensão induzida pela gravidez/ prevenção \& controle

Pré-eclâmpsia

Eclampsia

Keywords

Maternal mortality

Hypertension, pregnancy-induced/ prevention \& control

Pre-eclampsia Eclampsia

\section{Resumo}

OBJETIVO: identificar o perfil, a tendência e os determinantes da mortalidade materna por pré-eclâmpsia/eclâmpsia no estado do Paraná. MÉTODOS: estudo descritivo, de corte transversal sobre a mortalidade materna por pré-eclâmpsia/ eclâmpsia, no período entre 1997 e 2005. Os dados foram obtidos dos estudos de caso elaborados pelos Comitês de Mortalidade Materna, que utilizam o método Reproductive Age Mortality Survey para investigação de todos os óbitos de mulheres em idade fértil. Foram calculadas a razão de mortalidade materna (RMM) geral e a específica por pré-eclâmpsia/eclâmpsia. Para avaliar a tendência, os triênios foram comparados dois a dois considerando-se a estimação da RMM em cada triênio $(p<0,05)$. Foram analisados 56 óbitos por pré-eclâmpsia/eclâmpsia do triênio 2003 a 2005. As variáveis analisadas foram idade, renda, escolaridade, número e complicações nas gestações, condições do pré-natal, sinais e sintomas relacionados ao agravo, via de parto, tempo de interrupção da gestação, condições do recém-nascido, acesso e tratamento, evitabilidade e medidas de prevenção. RESULTADOS: a RMM geral por triênio apresentou tendência significativa de redução, chegando a 64,3/100.000 nascidos vivos. Houve estabilidade ao longo do tempo na RMM por transtornos hipertensivos, com RMM de 1 1,8/100.000 nascidos vivos. As mulheres acima de 40 anos e com baixo status socioeconômico apresentaram maiores riscos às primigestas. Constatou-se, com relação ao tratamento, subutilização ou o uso inadequado de medicamentos consagrados no tratamento da pré-eclâmpsia grave e da eclâmpsia. A análise dos comitês apontou que todos os óbitos maternos por este agravo poderiam ter sido evitados. CONCLUSÕES: recomenda-se a implementação de ações voltadas à minimização do conjunto de determinantes dos óbitos por pré-eclâmpsia no Paraná, incluindo a capacitação e o monitoramento dos profissionais de saúde para aplicação dos protocolos de tratamento e a formalização de uma rede de referência ambulatorial e hospitalar qualificada para atendimento à gestação de alto risco e suas intercorrências, com vinculação das gestantes no pré-natal.

\section{Abstract}

PURPOSE: to identify the profile, tendency and causes of maternal death by pre-eclampsia/eclampsia in Paraná. METHODS: descriptive, transversal cohort study on maternal death by pre-eclampsia/eclampsia from 1997 to 2005. Data were obtained from case studies prepared by Maternal Death Committees that employ the Reproductive Age Mortality Survey Method to examine all the cases of death among women in fertile age. The general and specific maternal death rate (MDR) by pre-eclampsia/eclampsia were considered. To evaluate the tendency, triennial periods have been compared, two by two, taking into consideration the MDR of each period ( $p<0.05$ ). In the triennial period from 2003 to 2005, 56 deaths by pre-eclampsia/eclampsia were analyzed. The variables focused were: age, income, schooling, gestation number and complications, pre-natal conditions, signs and symptoms related to the condition, delivery route, the time gestation was interrupted, the newborn conditions, access and treatment, ability to avoid and prevention measures. RESULTS: the general triennial MDR has presented significant decline, with $64.3 / 100,000$ bornalive babies. There has been stability along the period for MDR by hypertensive disorder, with MDR of 1 1.8/100,000 born-alive. Primiparous women, women over 40 and with low socio-economical status have presented higher risks. In relation to the treatment, there has been underuse or inadequate use of conventional medicines for severe pre-eclampsia and eclampsia. The committees' analysis indicated that all the maternal death due to these conditions could have been
Correspondência:

Vânia Muniz Néquer Soares Rua Plácido Prevedello, 266 - Ahú CEP 82200-420 - Curitiba (PR), Brasil E-mail: vaniam@onda.com.br

Recebido 3/8/09

' Professora-assistente da Universidade Tuiuti do Paraná; Membro do Comitê Estadual de Mortalidade Materna da Secretaria Estadual de Saúde do Paraná - Curitiba (PR), Brasil.

2 Departamento de Enfermagem Materno-Infantil e Saúde Pública da Escola de Enfermagem da Universidade Federal de Minas Gerais - UFMG - Belo Horizonte (MG), Brasil.

${ }^{3}$ Enfermeira Obstetra da Maternidade Dona Catarina Kuss; Secretaria de Estado da Saúde de Santa Catarina - Mafra (SC), Brasil. ${ }^{4}$ Enfermeira Obstetra da Maternidade Dona Catarina Kuss; Secretaria de Estado da Saúde de Santa Catarina - Mafra (SC), Brasil. ${ }^{5}$ Enfermeira Obstetra da Maternidade Dona Catarina Kuss; Secretaria de Estado da Saúde de Santa Catarina - Mafra (SC), Brasil. 
avoided. CONCLUSIONS: actions aiming at minimizing the set of causes that lead to death by pre-eclampsia in Paraná should be enforced, including the training and monitoring of health professionals in order to apply the treatment protocols, besides the formalization of a reference net of clinics and hospitals, qualified for the care of high risk pregnancy and its intercurrences, to which pre-natal pregnant women are enrolled.

\section{Introdução}

A mortalidade materna, por sua dimensão e consequências negativas, tem sido considerada um problema crucial de saúde pública. Evitável em mais de $90 \%$ dos casos, constitui-se uma grave violação dos direitos reprodutivos e, portanto, dos humanos ${ }^{1,2}$. É um dos indicadores da assistência à saúde das mulheres e, por esta razão, revela a qualidade da atenção à saúde e, indiretamente, as condições de vida e saúde da população ${ }^{3,4}$.

A World Health Organization (WHO) assinala a ocorrência de aproximadamente 529.000 óbitos de mulheres por ano, relacionados à gravidez, parto, puerpério e aborto. Destes, menos de $1 \%$ ocorrem em países desenvolvidos, refletindo as desigualdades sociais e em saúde das mulheres entre países pobres e ricos ${ }^{5,6}$.

No Brasil, a hipertensão arterial na gravidez constitue a primeira causa de morte materna. Estudo recente apontou que, nas capitais brasileiras, os transtornos hipertensivos lideram as causas deste tipo de morte, representando em torno de $25 \%$ dos óbitos maternos investigados ${ }^{3}$.

A etiopatogenia da hipertensão arterial gestacional é desconhecida, bem como a patogênese de suas manifestações. Surge, em geral, na segunda metade da gestação, mormente após a $20^{a}$ semana. Pode ser precedida por edema visível ou oculto, revelado pelo aumento brusco do peso corporal e, ainda, por proteinúria e elevação da pressão arterial, além de 140/90 $\mathrm{mmHg}$ ou o aumento de $30 \mathrm{mmHg}$ para a pressão sistólica e de $15 \mathrm{mmHg}$ para a diastólica ${ }^{7,8}$.

Ressalta-se a importância de se delimitar os níveis da mortalidade materna em cada região. No entanto, apenas o cômputo dos óbitos e a correção de sua subnotificação não têm sido suficiente para revertê-los. É preciso também identificar e intervir nos fatores subjacentes que conduzem à morte materna ${ }^{6}$. Neste sentido, é imperioso insistir no detalhamento de cada óbito, reconhecer seus determinantes, de forma que suas análises permitam a reorganização de ações sociais e em saúde visando à superação da problemática responsável pela morte de mulheres em decorrência da gravidez, parto ou puerpério.

Este estudo tem como objetivo identificar o perfil, tendência e possíveis determinantes da mortalidade materna por pré-eclâmpsia/eclâmpsia, no estado do Paraná, Brasil, no período de 1997 a 2005, com o detalhamento dos determinantes dos óbitos maternos por estas causas no período de 2003 a 2005.

\section{Métodos}

Estudo descritivo, de corte transversal, que apresenta a avaliação das tendências da mortalidade materna geral e por pré-eclâmpsia/eclâmpsia, no Paraná, entre 1997 e 2005, subdividido nos três triênios, 1997 a 1999, 2000 a 2002 e 2003 a 2005. Para tanto, foram calculadas a razão de mortalidade materna (RMM) geral e a específica por préeclâmpsia/eclâmpsia, com detalhamento dos óbitos maternos por estes agravos ( $n=56$ ), ocorridos entre 2003 e 2005.

A RMM geral foi obtida por meio da relação entre o número de óbitos de mulheres por complicações da gravidez, parto e puerpério até 42 dias após o parto, e o número de nascidos vivos (NV) expresso por $100.000 \mathrm{NV}$. A RMM por hipertensão arterial resultou da razão entre o número de óbitos maternos causados pela doença e o número de NV, também expressos por 100.000 NV. Foram excluídos os óbitos maternos tardios, que correspondem às mortes que ocorrem após o $42^{\circ}$ dia do puerpério até um ano após o parto.

Os dados dos óbitos foram extraídos do banco de dados e dos Estudos de Caso de Óbitos Maternos elaborados pelo Comitê Estadual de Prevenção da Mortalidade Materna (CEPMM/PR). Os dados sobre o número de NV foram obtidos do Sistema de Informações sobre NV.

Os Comitês de Prevenção da Mortalidade Materna (municipais, regionais e estadual) atuam no Paraná desde 1989. Estes Comitês realizam a vigilância epidemiológica sistemática dos óbitos maternos, identificando a real magnitude e corrigindo a subnotificação destas mortes. A investigação dos óbitos é realizada pelo método Reproductive Age Mortality Survey (RAMOS), que inclui a investigação de todos os óbitos de mulheres em idade fértil, mediante pesquisa em registros de prontuários hospitalares e ambulatoriais, entrevistas domiciliares com os familiares e profissionais que atenderam o caso, e laudos de necropsia, entre outros documentos disponíveis 9 . Desta forma, as informações da declaração de óbito original (DO-O) são complementadas, permitindo análise acurada das mortes ${ }^{10}$.

As informações obtidas nessas investigações são inseridas em banco de dados e sintetizadas em estudos de caso individuais. Posteriormente, são analisadas em câmaras técnicas multiprofissionais e interinstitucionais, as quais identificam a causa básica do óbito, os fatores que levaram ao óbito, a evitabilidade e as medidas de prevenção?.

As variáveis obtidas destas fontes e analisadas na presente pesquisa foram: idade, renda e escolaridade, 
número de gestações, complicações na gestação atual e anteriores, condições do pré-natal (número de consultas, idade gestacional no início e no término, idade gestacional no internamento), os sinais e sintomas apresentados, a via de parto, o tempo de interrupção da gestação e as condições do recém-nascido (RN), o acesso e a adequabilidade da assistência e do tratamento, a evitabilidade e as medidas de prevenção dos óbitos. Foi calculado, ainda, o risco relativo para as variáveis idade e escolaridade, das quais foi possível obter a população específica.

Os estudos de caso são de domínio público e têm sido amplamente divulgados dentro e fora do Estado, preservando-se; no entanto, o anonimato das mulheres, profissionais e serviços envolvidos. A utilização desses dados foi autorizada pelo Departamento de Vigilância Epidemiológica (DEVE) da Secretaria Estadual de Saúde do Paraná (SESA/PR).

Os resultados obtidos no estudo foram expressos por frequências e percentuais, razões de mortalidade e riscos relativos. Para avaliar a existência de tendência, os triênios foram comparados dois a dois considerando-se, para tanto, a estimação da razão de mortalidade em cada um desses triênios. Os valores de $\mathrm{p}<0,05$ indicaram significância estatística. Os dados foram organizados e analisados em planilha Excel.

\section{Resultados}

Ocorreram, no total, 190 mortes maternas por préeclâmpsia/eclâmpsia entre 1997 e 2005, representando $18 \%$ dos óbitos maternos, constituindo a primeira causa de mortalidade materna no estado do Paraná (Tabela 1).

Para análise da tendência da RMM geral, os triênios foram comparados dois a dois em relação à RMM. Em cada comparação testou-se a hipótese nula de RMM iguais nos dois triênios sob comparação, versus a hipótese alternativa de RMM diferentes.

$\mathrm{Na}$ comparação entre os primeiros dois triênios, ou seja, 1997 a 1999 e 2000 a 2002, observaram-se a RMM de 77,7 por $100.000 \mathrm{NV}$ e de 63,1 por 100.000 $\mathrm{NV}$, respectivamente. Estes resultados indicaram redução significativa na RMM ( $<<0,001)$.

Quando foram comparados os triênios 2000 a 2002 e 2003 a 2005, verificou-se RMM de 63,1 por 100.000 naquele, enquanto neste, a RMM passou para 64,2 por
100.000. Este resultado não indicou ter havido redução significativa na RMM ( $\mathrm{p}=0,810)$. No entanto, a comparação entre os triênios 1997 a 1999 e 2003 a 2005 apontou para uma redução significativa da RMM ( $\mathrm{p}=0,001)$. Sendo assim, em termos de avaliação da tendência, houve melhora significativa dos dois últimos triênios em relação ao primeiro.

$\mathrm{Na}$ análise de tendência da RMM por pré-eclâmpsia/ eclâmpsia, os triênios também foram confrontados dois a dois. Da mesma forma, testou-se a hipótese nula de RMM por pré-eclâmpsia/eclâmpsia para cada 100.000 NV iguais nos dois triênios sob comparação versus a hipótese alternativa de razões diferentes.

Estas indicaram não ter havido diferença significativa na razão de mortalidade por pré-eclâmpsia/eclâmpsia para cada 100.000 NV ( $\mathrm{p}=0,6)$ entre os dois primeiros triênios, tendo em vista que no triênio de 1997 a 1999 foi observada razão de 13,1 por 100.000 , enquanto no triênio 2000 a 2002 esta razão passou para 11,7 por 100.000 NV.

O mesmo ocorreu quando confrontados os triênios 2000 a 2002 e 2003 a 2005, isto é, não houve diferença significativa na razão de mortalidade por pré-eclâmpsia/ eclâmpsia para cada 100.000 NV ( $\mathrm{p}=0,9)$. Entre 2000 e 2002, observou-se razão de 11,7 por $100.000 \mathrm{NV}$, enquanto que no triênio 2003 a 2005 esta razão passou para 11,8 por $100.000 \mathrm{NV}$. A comparação entre os triênios 1997 a 1999 e 2003 a 2005 também não indicou diferença significativa na razão de mortalidade por pré-eclâmpsia/ eclâmpsia para cada $100.000(\mathrm{p}=0,630)$. Sendo assim, em termos de avaliação da tendência, houve estabilidade ao longo do tempo na razão de mortalidade por transtornos hipertensivos.

Com o detalhamento dos óbitos por pré-eclâmpsia/ eclâmpsia no triênio 2003 a 2005, verificou-se que, das 56 mulheres, 48,2\% encontravam-se na faixa etária de 20 a 29 anos, a qual também concentrou o maior número de nascimentos, determinando RMM de 10 por $100.000 \mathrm{NV}$. As mulheres com 40 anos ou mais apresentaram risco relativo 5,6 vezes mais elevado (RMM 60,51); observou-se o menor risco entre as adolescentes (Tabela 2).

As mulheres que morreram por pré-eclâmpsia/ eclâmpsia neste triênio apresentaram baixa escolaridade e renda. Os dados demonstraram que $66,1 \%$ delas tinham até oito anos de estudo incompletos e cerca de $59 \%$, renda familiar inferior a três salários mínimos. O risco de

Tabela 1 - Número de óbitos maternos, número de nascidos vivos, razão de mortalidade materna (RMM) geral e específica por pré-eclâmpsia/eclâmpsia no estado do Paraná (1997 a 2005)

\begin{tabular}{lcccccc}
\hline Triênio & Óbitos maternos (n) & Nascidos vivos (n) & RMM geral & $\begin{array}{c}\text { Óbitos por pré- } \\
\text { eclâmpsia/eclâmpsia (n) }\end{array}$ & $\begin{array}{c}\text { Óbitos por pré- } \\
\text { eclâmpsia/eclâmpsia (\%) }\end{array}$ & $\begin{array}{c}\text { RMM por pré-eclâmpsia/ } \\
\text { eclâmpsia }\end{array}$ \\
\hline $1997-1999$ & 440 & 566.650 & $77,7(73,0-82,3)^{\star}$ & 74 & 16,8 & $13,1(8,5-17,7)^{\star}$ \\
$2000-2002$ & 323 & 510.962 & $63,1(57,9-68,6)^{\star}$ & 60 & 18,3 & $11,7(8,1-15,4)^{\star}$ \\
$2003-2005$ & 306 & 476.183 & $64,2(57,6-70,9)^{\star}$ & 56 & 18,6 & $11,8(9,2-14,4)^{\star}$ \\
\hline
\end{tabular}

*limite inferior e limite superior: $p<0,05$ 
Tabela 2 - Perfil sociodemográfico dos óbitos maternos por pré-eclâmpsia/eclâmpsia no estado do Paraná (1997 a 2005)

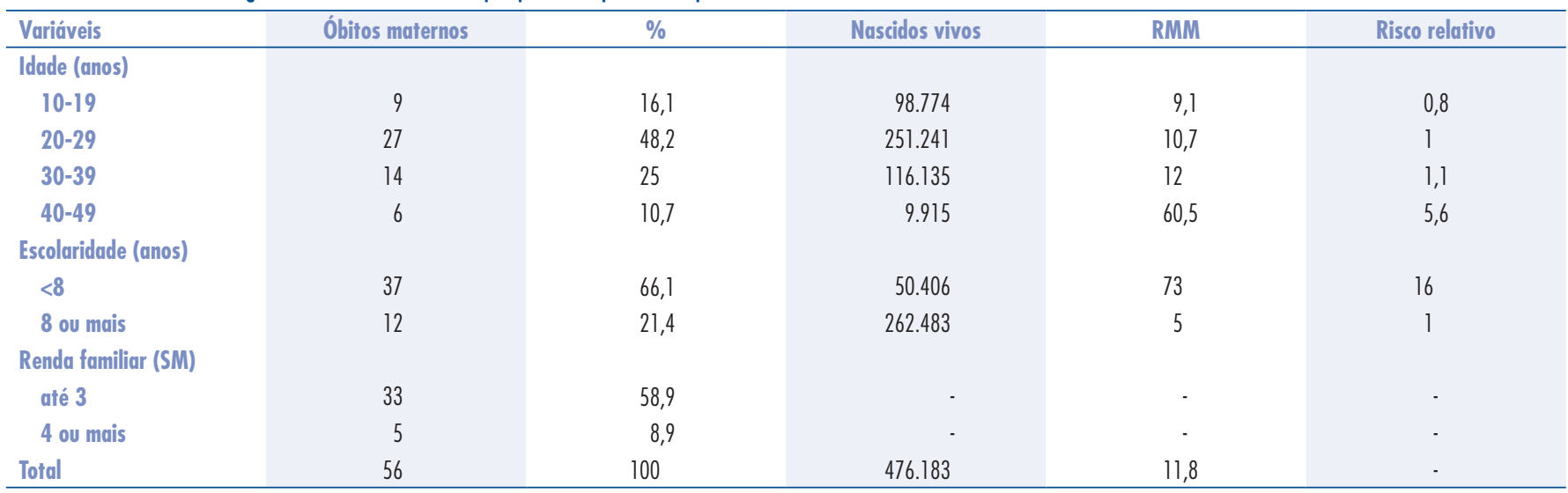

SM: salários mínimos; RMM: razão de mortalidade materna. *excluídos os ignorados (sete casos na variável anos de estudo e 18 casos na variável renda familiar).

morte materna foi 22 vezes maior para as mulheres sem nenhuma escolaridade (Tabela 2).

O perfil reprodutivo evidenciou maior proporção de óbitos entre as primigestas, 22 óbitos $(39,3 \%)$, seguidas das multigestas $(\geq 4)$ com $15(26,8 \%)$. Dentre as complicações apresentadas nas gestações anteriores, a hipertensão arterial sistêmica (HAS), a pré-eclâmpsia/eclâmpsia e as hemorragias foram as mais frequentes.

As intercorrências identificadas na gestação, em que ocorreu o óbito, durante o período pré-natal, além da pré-eclâmpsia foram: HAS, obesidade, ganho de peso ponderal acima do esperado, infecção do trato urinário, pielonefrite e gemelaridade (dados não-tabulados).

$\mathrm{Na}$ Tabela 3 verificou-se que $57,1 \%$ das gestantes frequentaram pré-natal com seis ou mais consultas e $8,9 \%$ não tiveram acompanhamento médico durante a gestação. Das que foram atendidas no pré-natal, 51,8\% iniciaram precocemente, antes da $14^{a}$ semana de gestação. No entanto, em $53,6 \%$ dos casos a última consulta ocorreu antes da $37^{a}$ semana de gestação, podendo indicar alta precoce do pré-natal ou internamento antecipado devido às complicações da hipertensão.

Os principais sinais e sintomas apresentados no momento da internação, além da elevação da pressão arterial (PA) foram: edema (34\%), cefaleia (20\%) e náuseas/vômitos $(16 \%)$, além de escotomas e dor epigástrica.

A via de parto predominante foi a cesárea $(75 \%$ dos casos) e a interrupção da gravidez ocorreu entre uma a quatro horas após a internação para 30,4\% das mulheres. Com relação às condições do $\mathrm{RN}$ no primeiro minuto de vida, verificou-se que $21(38 \%)$ nasceram vivos; destes, metade apresentou índice de Apgar acima de sete e os outros 19 (34\%) tiveram índice menor que sete. Em 12 casos $(21 \%)$ ocorreu o óbito fetal durante a gestação e em quatro $(7 \%)$ não constava esta informação.

A causa básica dos óbitos foi a pré-eclâmpsia (CID O14) em 46,4\% dos óbitos (26 casos). A eclâmpsia (CID
Tabela 3 - Perfil do pré-natal nos casos de óbitos maternos por pré-eclâmpsia/eclâmpsia no estado do Paraná (1997 a 2005)

\begin{tabular}{|c|c|c|}
\hline Variáveis* & n & $\%$ \\
\hline \multicolumn{3}{|c|}{ Número de consultas de pré-natal } \\
\hline$<6$ & 14 & 25 \\
\hline 6 ou mais & 32 & 57,1 \\
\hline Não realizou & 5 & 8,9 \\
\hline \multicolumn{3}{|c|}{ Idade gestacional na primeira consulta de pré-natal** } \\
\hline $1-13$ semanas & 30 & 51,8 \\
\hline $14-26$ semanas & 10 & 19,6 \\
\hline $27-40$ semanas & 1 & 1,8 \\
\hline \multicolumn{3}{|c|}{ Idade gestacional na última consulta de pré-natal** } \\
\hline até 30 semanas & 9 & 16,1 \\
\hline 31 a 36 semanas & 21 & 37,5 \\
\hline 37 a 40 semanas & 8 & 14,3 \\
\hline Total & 56 & 100 \\
\hline
\end{tabular}

*excluídos os ignorados; * excluídas as que não realizaram pré-natal.

O15) foi confirmada em 39,3\% das mortes (22 casos). Analisando o período em que ocorreram as convulsões, identificou-se que a maioria, 16,1\% (nove casos), ocorreu na gestação (CID O15.0); 7,1\% (quatro casos) durante o trabalho de parto ou parto (CID 15.1); $12,5 \%$ (sete casos) aconteceram no período pós-parto (CID O15.2); 3,6\% (dois casos) não havia especificação quanto ao período de ocorrência. A pré-eclâmpsia superposta à HAS (CID O11) ocorreu em 14,3\% dos óbitos (oito casos).

Considerando as condutas e o tratamento para a complicação, identificou-se que apenas $46,4 \%$ das mulheres receberam o medicamento sulfato de magnésio $\left(\mathrm{MgSO}_{4}\right)$, ou seja, 26 das 56 mulheres. É importante ressaltar que o uso da medicação, em alguns casos, não ocorreu no tempo oportuno, segundo análise nas câmaras técnicas dos Comitês.

Verificou-se a utilização de uma grande variedade de outros anti-hipertensivos, entre eles a metildopa em 36 casos; nefedipina em 26; captopril em seis e a hidralazina 
em quatro. Foi utilizada, ainda, uma diversidade de tranquilizantes e anticonvulsivantes como diazepan, em 14 casos; levopromazina em 15; hidantoína em quatro e haloperidol em dois. Foram ministrados hemoderivados em 49 casos e antibióticos em 29.

Necessitaram e conseguiram transferência $57 \%$ das mulheres; em $9 \%$ dos casos a transferência não foi possível por falta de vaga. Das pacientes transferidas, $81 \%$ foram para uma Unidade de Terapia Intensiva (UTI) no mesmo ou em outro hospital.

A pré-eclâmpsia/eclâmpsia encontravam-se subnotificada em $23 \%$ das declarações de óbito, ou seja, 13 casos foram acrescentados aos 43 declarados, após a investigação realizada pelos Comitês. As causas que ocultavam estas mortes na declaração de óbito (DO) foram, principalmente, $o$ acidente vascular cerebral e a embolia.

Após análise detalhada de cada óbito materno nas câmaras técnicas dos Comitês, concluiu-se que todas as mortes poderiam ter sido evitadas, sendo por melhor diagnóstico no pré-natal (67,9\% dos casos), acesso a tratamento adequado em serviços especializados no prénatal $(60,7 \%)$, vigilância e busca ativa dos casos de risco gestacional no pré-natal $(51,8 \%)$ e, principalmente, melhor diagnóstico e tratamento desta complicação em serviço hospitalar especializado ( $97 \%$ dos casos).

Quando a distribuição dos óbitos é situada, segundo as 22 regiões de saúde (divisão administrativa adotada pela Secretaria Estadual de Saúde do Paraná), constatou-se que a maioria, ou 16 casos, ocorreu na Segunda Regional, a qual engloba a capital e sua região metropolitana. Somente em três regionais não ocorreram óbitos por hipertensão na gestação nestes três anos.

\section{Discussão}

O estudo da tendência e dos determinantes da mortalidade materna é importante para demonstrar a situação da assistência à saúde de uma região ou comunidade, bem como para propor ações concretas para a redução deste evento ${ }^{3}$.

O Paraná apresenta RMM geral com tendência significativa à redução, mas é ainda elevada (65/100.000) se comparada a de países desenvolvidos, nos quais a média da RMM não ultrapassa a 10 por $100.000^{5}$.

Os transtornos hipertensivos na gestação, que constituem a primeira causa de morte materna no Estado, não apresentaram tendência significativa de redução entre os triênios estudados. Pode-se deduzir que esse fato está relacionado a dificuldades de acesso a serviços especializados e à qualidade da assistência prestada. O Paraná conta com ampla rede de Unidades Básicas de Saúde, além de 298 hospitais/maternidades para gestantes de baixo risco e 29 serviços hospitalares classificados com nível secundário e terciário para referência a gestantes de alto risco. As mulheres tiveram acesso a atendimento nestes serviços ( $57 \%$ das mulheres que foram a óbito tiveram mais de seis consultas de pré-natal, e em $100 \%$ dos casos o parto foi hospitalar, $57 \%$ foram transferidas a serviços de maior complexidade e a maioria teve acesso à UTI); no entanto, foram pouco resolutivos.

Estudo sobre mortes maternas relacionadas às complicações da hipertensão arterial, no período de 1995 a 1999, realizado no município de São Paulo, identificou falhas semelhantes no atendimento às mulheres, entre as quais: planejamento familiar deficiente; assistência prénatal inadequada e ineficiente; falta de vaga no serviço hospitalar; deficiência de estrutura dos hospitais, acarretando precariedade no atendimento às gestantes com hipertensão e complicações decorrentes deste agravo ${ }^{4}$.

No referido estudo, a hipertensão na gestação foi responsável por 23\% dos 609 óbitos ocorridos em São Paulo naquele período (142 óbitos), incluindo a HAS, que resultou em uma RMM de 13,2 por $100.000 \mathrm{NV}$. A pré-eclâmpsia/eclâmpsia liderou as causas de morte por hipertensão com 99 casos (RMM de 9,21) e 43 foram devido à hipertensão sistêmica ou crônica na gestação. A RMM por pré-eclâmpsia/eclâmpsia do Paraná $(11,8)$ foi superior à encontrada para o município de São Pauló ${ }^{4}$.

Foram observadas dificuldades e demora quanto ao tratamento e às transferências para serviços de referência, resultando que as mulheres chegavam a estes, muitas vezes, em estado crítico. Quanto ao atendimento, verificou-se a inexistência da homogeneidade de condutas no atendimento emergencial; a utilização de uma multiplicidade de drogas visando o controle pressórico é um indicativo desta afirmação.

Constatou-se a subutilização, ou mesmo o uso de forma inadequada, de medicamentos de uso consagrado no tratamento da pré-eclâmpsia grave e da eclâmpsia, como o sulfato de magnésio e a hidralazina. A subutilização desses medicamentos no combate às convulsões e hipertensão, identificada pelos Comitês, é preocupante e permite o questionamento quanto às condutas dos profissionais e das instituições hospitalares ${ }^{11}$, na medida em que as recomendações e os protocolos disponíveis do Ministério da Saúde e da Federação Brasileira de Ginecologia e Obstetrícia (FEBRASGO) e pela literatura internacional não são seguidos ou considerados.

A presença de um profissional habilitado na assistência ao parto é essencial, no entanto no caso brasileiro não tem sido o suficiente, sobretudo devido à falta de adesão a protocolos baseados em evidências para o manejo de gravidezes de alto risco. No estudo realizado na cidade de São Paulo, menos de $10 \%$ das mulheres que morreram por eclâmpsia (em hospitais e atendidas por médicos) foram tratadas com o sulfato de magnésio; outras drogas 
utilizadas para as convulsões e controle das complicações foram fenobarbital, pindolol, hidralazina, metildopa, propanolol, nifedipina, benzodiazepínicos e diuréticos ${ }^{4}$. No Paraná o uso destes medicamentos foi observado, mas a proporção de casos que se utilizou o sulfato de magnésio foi superior (50\%) em relação à do município de São Paulo. Isto denota possivelmente o uso incorreto desta medicação, uma vez que o desfecho foi a morte materna.

Uma equipe multiprofissional treinada e capacitada; disponibilidade de equipamentos e infraestrutura laboratorial adequados ao diagnóstico e tratamento dos casos de pré-eclâmpsia/eclâmpsia e das gestações de alto risco, em geral, são indispensáveis. A oferta de atenção qualificada aparece como um componente essencial para a redução da mortalidade materna ${ }^{12}$.

Algumas mulheres deste estudo tiveram alta sem acompanhamento posterior e retornaram em estado grave. Após a alta hospitalar, as puérperas, de modo geral, deveriam ter acesso à consulta de puerpério e, em particular, as que tiveram pré-eclâmpsia/eclâmpsia ou HAS. Torna-se imperioso o monitoramento da continuidade do tratamento anti-hipertensivo, além da identificação de risco reprodutivo e aconselhamento para o uso de métodos contraceptivos adequados, com base em informações seguras e de qualidade ${ }^{7}$.

Foi observado um risco quase seis vezes mais elevado para as mulheres com mais idades para morte por préeclâmpsia/eclâmpsia. A RMM foi menos elevada para as adolescentes, embora numérica e proporcionalmente fossem superiores ao das mulheres com 40 anos. Além dos fatores negativos relacionados à gravidez na adolescência, como baixa escolaridade, ausência de consultas ginecológicas prévias e falta de acesso aos métodos anticoncepcionais ${ }^{13}$, a pré-eclâmpsia/eclâmpsia se constitui como uma das causas mais importantes da mortalidade de adolescentes grávidas demonstrada em outros estudos ${ }^{14}$.

$\mathrm{O}$ estresse, geralmente presente nas gestações indesejadas, frequentes nos extremos da vida reprodutiva, é apontado como fator de facilitação da pré-eclâmpsia e suas complicações ${ }^{15}$. Melhores condições dos serviços de planejamento familiar poderiam ter evitado parte destes óbitos. Os dados referentes à paridade apontaram maior proporção de óbitos entre as primigestas.

Os fatores de risco para pré-eclâmpsia/eclâmpsia apontados na literatura, dentre eles obesidade, primiparidade, idades extremas, antecedentes familiares, história de pré-eclâmpsia ou eclâmpsia em gestações anteriores, gemelaridade e ganho excessivo de peso foram identificados nos casos estudados.

A eclâmpsia ocorreu em 39,3\% dos casos, podendo estar associada a atrasos no diagnóstico e nas condutas. Similarmente ao referido por pesquisadores, ocorreu mais frequentemente no período anteparto ${ }^{7,8}$, embora o número de casos no pós-parto também seja preocupante.
As cesáreas praticadas em grande número dos casos pesquisados não contribuíram para evitar os óbitos. Nos casos da pré-eclâmpsia grave, esse procedimento é indicado para preservar a vida da mãe e do feto ${ }^{7}$. Neste estudo ocorreram casos em que no aguardo da viabilidade/ maturidade fetal houve agravamento do quadro clínico, impossibilitando a preservação da vida materna, indicando que o tempo determinado para interromper a gravidez pode ter sido inadequado. O período entre a internação e a interrupção da gestação variou de menos de 30 minutos a mais de 15 horas, com maior proporção ocorrendo entre uma e quatro horas após a internação.

Na emergência hipertensiva, é de fundamental importância a realização de uma criteriosa avaliação obstétrica e laboratorial, levando-se em conta os diversos fatores de interferência e prognósticos maternos e fetais, para então decidir o melhor momento da interrupção da gestação. Uma segunda opinião de médicos poderia dar maior segurança ao profissional no manejo da patologia e do parto ${ }^{7}$.

$\mathrm{Na}$ casuística deste estudo, além dos 56 óbitos maternos, foram apurados 12 óbitos fetais, mostrando a gravidade dos casos e prováveis falhas na condução da patologia. A avaliação do índice de Apgar dos recém-nascidos vivos mostrou que 19 neonatos (34\%) nasceram em condições que variaram entre regulares e más.

Para cerca de $70 \%$ dos casos, as internações ocorreram quando a idade gestacional correspondia a 31 semanas, período em que haveria viabilidade fetal, e cujas condições do feto dependeriam das condutas nas complicações maternas. Estudo realizado em uma maternidade-escola do Nordeste brasileiro demonstrou que $75,5 \%$ dos internamentos por hipertensão ocorreram neste período, observando que, como em outros estudos, as complicações da hipertensão na gestação ocorrem em sua maioria mais tardiamente; portanto, em uma época de comprovada viabilidade fetal ${ }^{16}$.

Todas as gestantes hipertensas investigadas receberam atendimento hospitalar, mas este ou foi tardio ou pouco resolutivo. A maioria necessitou de transferências após complicações. Falhas referentes ao acesso das gestantes à assistência obstétrica no momento do parto e no caso de complicações, levando-as à peregrinação em busca de um atendimento adequado, demonstram a desarticulação entre o sistema de referência e de contrarreferência, bem como seus efeitos negativos na qualidade do atendimento, particularmente em situações de risco ${ }^{17}$.

Os óbitos por pré-eclampsia/eclâmpsia ocorreram em quase todas as Regionais de Saúde, com maior número de casos ocorrendo na Segunda Regional de Saúde (Curitiba e Região Metropolitana), onde justamente se concentra a maior proporção de gestantes do Estado (30\%) e de serviços de referência. Faz-se necessária uma avaliação da RMM por RS e um monitoramento permanente das 
mortes por pré-eclâmpsia/eclâmpsia por regional e municípios, pois são causas evitáveis se detectadas e tratadas adequadamente.

A mortalidade materna por hipertensão na gestação foi controlada na maioria dos países desenvolvidos. No entanto, estudo realizado nos Estados Unidos identificou estagnação das taxas de mortalidade materna no país, embora em níveis inferiores aos observados no Brasil, sendo suas principais causas o tromboembolismo, as hemorragias e, em terceiro lugar, a hipertensão e suas complicações ${ }^{18}$.

No Brasil e no estado no Paraná, os transtornos hipertensivos, embora controláveis e evitáveis, continuam sendo a primeira causa de morte materna ${ }^{19}$ e um grave problema de saúde que ainda atinge significativo número de mulheres. É possível inferir que os determinantes da mortalidade materna por hipertensão na gestação, no Paraná, estão relacionados aos cuidados inadequados e às dificuldades de acesso a serviços especializados de referência para gestação de alto risco no pré-natal e na atenção ao parto e ao puerpério, de forma mais qualificada e resolutiva.

Por fim, verificou-se uma elevada subnotificação desta causa de morte nas declarações de óbitos, demonstrando a necessidade de treinamento para o correto preenchimento desse importante e imprescindível documento para as áreas da saúde, demográfica e jurídica.

Algumas recomendações se mostram pertinentes, tais como: regulamentação e monitoramento pelos gestores do Sistema Único de Saúde (SUS) e da Agência Nacional de Saúde Suplementar (ANS), da utilização de protocolos de tratamento já consagrados cientificamente e tecnicamente nos serviços públicos de saúde e dos planos privados de saúde, bem como proporcionar treinamentos e recursos para sua efetiva execução, com o apoio das universidades e dos serviços que atendem gestantes de risco.

Recomenda-se a implantação dos Comitês Hospitalares de Estudo e Prevenção da Morbimortalidade Materna e Neonatal, na medida em que estes têm a função de avaliar a assistência e os óbitos, especialmente nos casos de near miss (casos graves), identificando e corrigindo possíveis falhas, e observando o cumprimento dos protocolos do Ministério da Saúde ${ }^{20}$.

O estudo contribui para evidenciar a magnitude e os determinantes das mortes decorrentes dos distúrbios hipertensivos durante a gestação, no estado do Paraná, Brasil, incluindo a constatação de lenta redução das mortes por este agravo. São ressaltados, como condicionantes das mortes estudadas, os aspectos relativos à atenção em saúde, a inadequação da assistência prestada e a dificuldade de acesso a serviços de referência, além das condições sociais desfavoráveis conforme classificação proposta pela $\mathrm{WHO}^{6}$. Esta situação é confirmada pelo Comitê Estadual de Prevenção da Mortalidade Materna do Paraná (CEPMM/PR), ao concluir que todas as mortes maternas devido à pré-eclâmpsia/eclâmpsia poderiam ter sido evitadas. Ratifica-se a importância das ações multiprofissionais e multissetoriais voltadas à minimização do conjunto de determinantes que têm marcado o perfil da mortalidade materna, em geral e, especialmente, por pré-eclâmpsia/eclâmpsia, como descritas neste estudo.

\section{Referências}

1. Organização Mundial da Saúde. Promovendo a maternidade segura através dos direitos humanos. Rio de Janeiro: Cepia; 2003.

2. Cook RJ, Dickens BM, Fathalla MF. Saúde reprodutiva e direitos humanos: integrando medicina, ética e direito. Rio de Janeiro: Cepia \& Oxford; 2004

3. Laurenti R, Jorge MHPM, Gotlieb SLD. A mortalidade materna nas capitais brasileiras: algumas características e estimativa de um fator de ajuste. Rev Bras Epidemiol. 2004;7(4):449-60.

4. Vega CE, Kahhale S, Zugaib M. Maternal mortality due to arterial hypertension in São Paulo City (1995-1999). Clinics (Sao Paulo). 2007;62(6):679-84.

5. Maternal mortality in 2000: estimates developed by WHO, UNICEF and UNFPA [document on the Internet]. Geneva: WHO; 2004 [cited 2007 Jun 29]. Available from: http://www.reliefweb.int/ library/documents/2003/who-saf-22oct.pdf

6. World Health Organization. Beyond the numbers: reviewing maternal deaths and complications to make pregnancy safer [document on the Internet]. Geneva: WHO; 2004 [cited 2007 Jun 23].
Available from: http://whqlibdoc.who.int/publications/2004/ 9241591838.pdf

7. Ministério da Saúde. Gestação de alto risco: manual técnico. 4a ed. Brasília, DF: Ministério da Saúde; 2000. p. 21-30.

8. Sibai BM. Diagnosis, prevention, and management of eclampsia. Obstet Gynecol. 2005; 105(2):402-10. Review.

9. Soares VMN, Martins AL. Trajetória e experiência dos Comitês de Prevenção da Mortalidade Materna do Paraná. Rev Bras Matern Infant. 2006;6(4):453-60.

10. Laurenti R, Mello Jorge MHP, Gotlieb SLD. Mortes maternas e mortes por causas maternas. Epidemiol Serv Saúde. 2008;17(4): 283-92.

11. Duley L, Gülmezoglu AM, Henderson-Smart DJ. Magnesium sulphate and other anticonvulsants for women with pre-eclampsia. Cochrane Database Syst Rev. 2006(3):CD000025.

12. Amaral E, Luz AG, Souza JPD. A morbidade materna grave na qualificação da assistência: utopia ou necessidade? Rev Bras Ginecol Obstet. 2007;29(9):484-9. 
13. Amorim MMR, Lima LA, Lopes CV, Araújo DKL, Silva JGG, César $L C$, et al . Fatores de risco para a gravidez na adolescência em uma maternidade-escola da Paraíba: estudo caso-controle. Rev Bras Ginecol Obstet. 2009;31 (8):404-10.

14. Santos ZMSA, Neves MG, Nascimento JC, Fernandes HT, Feitoza JS. Autocuidado da gestante adolescente na prevenção dos fatores de risco da síndrome hipertensiva específica da gravidez (SHEG). Rev Bras Promoção Saúde. 2007;20(3): 173-80.

15. Takiuti NH, Kahhale S, Zugaib M. Stress in pregnancy: a new Wistar rat model for human preeclampsia. Am J Obstet Gynecol. 2002; 186(3):544-50
16. Bezerra EHM, Alencar Júnior CA, Feitosa RFG, Carvalho AAA. Mortalidade materna por hipertensão: índice e análise de suas características em uma maternidade-escola. Rev Bras Ginecol Obstet. $2005 ; 27(9): 548-53$.

17. Tanaka ACA. Uma aula e muitas lições sobre a mortalidade materna. J Rede Fem Saúde. 2006;28:7-9.

18. Lang CT, King JC. Maternal mortality in the United States. Best Pract Res Clin Obstet Gynaecol. 2008;22(3):517-31.

19. Ministério da Saúde. Saúde Brasil 2007: uma análise da situação do Brasil. Brasília, DF: Ministério da Saúde; 2008.

20. Ministério da Saúde. Manual dos Comitês de Mortalidade Materna. 3a ed. Brasília, DF: Ministério da Saúde; 2009. 